\title{
An Indirect Model Reference Adaptive Fuzzy Control for SISO Takagi-Sugeno Model
}

\author{
Young-Wan Cho, Chang-Woo Park, Ki-Chul Lee, and Mignon Park
}

\begin{abstract}
In this paper, a parameter estimator is developed for the plant model whose structure is represented by the Takagi-Sugeno model. The essential idea behind the on-line estimation is the comparison of the measured state with the state of an estimation model whose structure is the same as that of the parameterized model. Based on the parameter estimation scheme, an indirect Model Reference Adaptive Fuzzy Control (MRAFC) scheme is proposed to provide asymptotic tracking of a reference signal for the systems with uncertain or slowly time-varying parameters. The developed control law and adaptive law guarantee the boundedness of all signals in the closed-loop system. In addition, the plant state tracks the state of the reference model asymptotically with time for any bounded reference input signal.
\end{abstract}

Keywords: fuzzy control, model reference adaptive fuzzy control, robust control

\section{Introduction}

Control systems are increasingly required to have high dynamical performance and robust behaviors, yet are expected to cope with more complex, uncertain and highly nonlinear dynamic processes. Along this increased process complexity is increased abstraction and uncertainty in the models and their mathematical representation. One significant approach in dealing with major changes and uncertainty in nonlinear dynamical processes is through intelligent modelling and control.

In some control tasks, the systems to be controlled have parameter uncertainty. Unless such parameter uncertainty is gradually reduced on-line by an appropriate adaptation or estimation mechanism, it may cause inaccuracy or instability for the control systems. In many other tasks, such as those in power systems, the system dynamics may have well known dynamics at the beginning, but experience unpredictable parameter variations as the control operation goes on. Without continuous redesign of the controller, the initially appropriate controller design may not be able to control the changing plant well.

The adaptive control has been a main theme in control engineering, and related researches are expected to continue. Classical adaptive control methods have two kinds of approaches: One is LMAC(Learning Model Adaptive Control) which performs on-line estimation of parameters and the other is MRAC(Model Reference Adaptive Control)[1][2]. Although sufficient systematic theories exist for the adaptive control for linear systems, existing adaptive techniques can treat a special class of nonlinear systems having linearly parameterizable dynamics. Another major problem is thereal-time application. Real-time control means that between two sampling times, the optimal solution for the action must be determined and applied. Often, the system is known, and there exist exact ways to determine the optimal action; however, the computational complexity

Manuscript received: July 15, 2000., Accepted: Dec. 26, 2000.

Young-Wan Cho, Chang-Woo Park, Ki-Chul Lee, Mignon Park: Dept. of Electronic Eng., Yonsei Univ.(cyw@yeics.yonsei.ac.kr/cwpark@ yeics.yonsei.ac.kr/kclee@yeics.yonsei.ac.kr/mignpark@bubble.yonse i.ac.kr)

※This work was supported by the brain Korea 21 Project. of the solution is too high, so it is merely a hypothetical possibility.

On the other hand, fuzzy control methods have become popular and are being used widely in many practical and industrial situations. Fuzzy control methods have advantages such as robustness, which have been demonstrated through industrial applications[6]. Fuzzy controllers are supposed to work in situations where there is a large uncertainty or unknown variation in plant parameters and structures. Generally, the basic objective of adaptive control is to maintain consistent performance of a system in the presence of uncertainty or unknown variation in plant parameters. Therefore, advanced fuzzy control should be adaptive[8].

In order to deal with the uncertainties of nonlinear systems, in the fuzzy control system literature, a considerable amount of adaptive control schemes have been suggested.[3][6]-[8] The main advantages of adaptive fuzzy control over nonadaptive fuzzy control are: 1) better performance is usually achieved because the adaptive fuzzy controller can adjust itself to the changing environment, and 2) less information about the plant is required because the adaptation law can help to learn the dynamics of the plant during real-time operation[8]. However, these approaches still have some problems. Maeda and Murakami's work[6] does not include systematic analysis such as stability and convergency. The adaptive gain scheduling control schemes for TS model such as Kim's[3] have weakness in parameter estimation which is needed for appropriate gain selection.

In this paper, an indirect Model Reference Adaptive Fuzzy Control (MRAFC) scheme is proposed to provide asymptotic tracking of a reference signal for the systems having uncertain or slowly time-varying parameters. In many applications, the structure of the model of the plant may be known, but its parameters may be unknown and/or change with time. To design the controller parameters for this kind of plant model, the parameters have to be estimated by observing the behavior of the system. This paper presents the design and analysis of on-line parameter estimator for the plant model whose structure is represented by the Takagi-Sugeno model. The essential idea behind the on-line estimation is the comparison of the measured state with the state of an estimation model whose structure is the 
same as that of the parameterized model. The adaptation law adjusts the controller parameters on-line so that the plant output tracks the reference model output. The developed control law and adaptive law guarantee the boundedness of all signals in the closed-loop system. In addition, the plant state tracks the state of the reference model asymptotically with time for any bounded reference input signal.

The proposed adaptive fuzzy control scheme is applied to tracking and regulating control of a inverted pendulum system to verify the validity and effectiveness of the control scheme.

\section{Takagi-Sugeno model based fuzzy control}

In system analysis such as stability, robustness and performance, it is important and significant to select an appropriate model representing a real system. From this viewpoint, among various kinds of fuzzy modeling methodologies, a fuzzy model called Takagi-Sugeno (TS) model[10] is widely accepted as a powerful tool for design and analysis of fuzzy control systems and applications of the TS models to various kinds of nonlinear systems can be found[14]. The Takagi-Sugeno type fuzzy model uses the smooth aggregation of local linear mathematical models to represent the dynamical systems, which are useful because they can provide description of a physical phenomenon or a process, and can be well suited to analysis, prediction and design of dynamic control system.

Consider the continuous-time nonlinear system described by the TS model. The $i$ th rule of continuous-time TS model is of the following form:

$$
\begin{aligned}
R^{i}: & \text { If } x_{1}(t) \text { is } M_{1}^{i} \text { and } \cdots \text { and } x_{n}(t) \text { is } M_{n}^{i} \\
& \text { then } \dot{\boldsymbol{x}}(t)=A_{i} \boldsymbol{x}(t)+B_{i} \boldsymbol{u}(t)
\end{aligned}
$$

where $\boldsymbol{x}^{T}(t)=\left[x_{1}(t), x_{2}(t), \cdots, x_{n}(t)\right]$,

$$
\boldsymbol{u}^{T}(t)=\left[u_{1}(t), u_{2}(t), \cdots, u_{m}(t)\right] .
$$

Given a pair of input $(\boldsymbol{x}(t), \boldsymbol{u}(t))$, the final output of the fuzzy system is inferred as follows:

$$
\dot{\boldsymbol{x}}(t)=\frac{\sum_{i=1}^{l} w_{i}(t)\left\{A_{i} \boldsymbol{x}(t)+B_{i} \boldsymbol{u}(t)\right\}}{\sum_{i=1}^{l} w_{i}(t)}
$$

where $w_{i}(t)=\coprod_{j=1}^{n} M_{j}^{i}\left(x_{j}(t)\right)$, and $M_{j}^{i}\left(x_{j}(t)\right)$ is the grade of membership of $x_{j}(t)$ in $M_{j}^{i}$.

In order to design fuzzy controllers to stabilize fuzzy system (2), we utilize the concept of parallel distributed compensation (PDC)[11]. The idea is to design a compensator for each rule of the fuzzy model. For each rule, we can use linear control techniques. The resulting overall controller, which is nonlinear in general, is a fuzzy blending of each individual linear controller. The PDC controller shares the same fuzzy sets with fuzzy model (2) to construct its premise part. That is, the PDC controller is of the following form:

$$
\begin{aligned}
& R^{i}: \text { If } x_{1}(t) \text { is } M_{1}^{i} \text { and } \cdots \text { and } x_{n}(t) \text { is } M_{n}^{i} \\
& \text { then } \boldsymbol{u}(t)=-K_{i} \boldsymbol{x}(t)
\end{aligned}
$$

where $x^{T}(t)=\left[x_{1}(t), x_{2}(t), \cdots, x_{n}(t)\right]$ and $i=1,2, \cdots, l$.

Given a state feedback $x(t)$, the final output of the fuzzy PDC controller (3) is inferred as follows:

$$
\boldsymbol{u}(t)=-\frac{\sum_{i=1}^{l} w_{i}(t) K_{i} \boldsymbol{x}(t)}{\sum_{i=1}^{l} w_{i}(t)}
$$

where $w_{i}(t)=\coprod_{j=1}^{n} M_{j}^{i}\left(x_{j}(t)\right)$

By substituting the controller (4) into model (2), we can construct the closed-loop fuzzy control system as following:

$$
\dot{\boldsymbol{x}}(t)=\frac{\sum_{l=1}^{l} \sum_{j=1}^{l} w_{i}(t) w_{j}(t)\left\{A_{i^{-}} B_{i} K_{j}\right\} \boldsymbol{x}(t)}{\sum_{i=1}^{l} \sum_{j=1}^{l} w_{i}(t) w_{j}(t)}
$$

A sufficient condition for ensuring the stability of the closed-loop fuzzy system (5) is given in Theorem 1, which is derived in [14].

Theorem 1: The equilibrium of a fuzzy control system (5) is asymptotically stable in the large if there exists a common positive definite matrix $P$ such that

$$
G_{i j}^{T} P+P G_{i j}=-Q_{i j}
$$

for all $i, j=1,2, \ldots, l$

where $G_{i j}=A_{i^{-}} B_{i} K_{j}$ and $Q_{i j}$ is a positive definite matrix.

Proof: The proof is given in [14].

The design problem of model based fuzzy control is to select $K_{j}(j=1,2, \ldots, l)$ such that stability conditions (6) in Theorem 1 are satisfied. It has long been recognized that there is a lack of a systematic procedure to find a common positive definite matrix $P$ to check the stability of fuzzy system. Most of the time a trial-and-error type of procedure has been used[15]. In [12], the common $P$ problem was solved efficiently via convex optimization techniques for LMI's (Linear Matrix Inequality). However, the fuzzy control (4) does not guarantee the stability of system in the presence of parameter uncertainty or unmodeled dynamics. Moreover, the design of the control parameters is not possible for the systems whose parameters are unknown. To overcome these drawbacks, in this research, an adaptive control scheme for the plant models whose structures are known but parameters unknown.

\section{Fuzzy feedback linearization control of TS model}

Recently, some studies have been reported on fuzzy feedback linearization controller (FFLC) which are based on TS model[4][5][16]. These approaches fully utilize the properties of TS model such as simplicity and effectiveness in describing nonlinear systems. This section briefly introduces the FFLC proposed in [4] to develop an indirect 
adaptive fuzzy control system for the TS model.

Let us consider the following nonlinear system described by the TS model

$$
\begin{array}{r}
R^{i}: \text { If } x_{1} \text { is } M_{1}^{i}\left(x_{1}\right) \text { and } \cdots \text { and } x_{p} \text { is } M_{p}^{i}\left(x_{p}\right) \\
\text { then } \dot{\boldsymbol{x}}(t)=A_{i} \boldsymbol{x}(t)+B_{i} u(t)
\end{array}
$$

where, $R^{i}(i=1,2, \cdots, l)$ denotes the $i$ th implication, $l$ is the number of fuzzy implications, $p(p<n)$ is the number of state variables used as the input of premise part of implications, $\dot{x}$ is the output from the $i$ th implication, and $\boldsymbol{x}=\left[x_{n}, x_{n-1}, \cdots, x_{1}\right]^{T}$ is the state vector, $u$ is the input variable, and $M_{j}^{i}(j=1,2, \cdots, p)$ are fuzzy sets partitioning the input space.

Given a pair of input $\boldsymbol{x}$ and $u$, the TS fuzzy model is inferred as follows:

$$
\dot{\boldsymbol{x}}(t)=\frac{\sum_{i=1}^{l} w_{i}(\boldsymbol{x}(t))\left\{A_{i} \boldsymbol{x}(t)+B_{i} u(t)\right\}}{\sum_{i=1}^{l} w_{i}(\boldsymbol{x}(t))}
$$

where, $w_{i}(\boldsymbol{x}(t))=\prod_{j=1}^{p} M_{j}^{i}(\boldsymbol{x}(t))$

$$
A_{i}=\left|\begin{array}{ccccc}
a_{n}^{i} & a_{n-1}^{i} & \cdots & a_{2}^{i} & a_{1}^{i} \\
1 & 0 & \cdots & 0 & 0 \\
& & \vdots & & \\
0 & 0 & \cdots & 1 & 0
\end{array}\right|, B_{i}=\left|\begin{array}{c}
b^{i} \\
0 \\
\vdots \\
0
\end{array}\right|
$$

In (10), it is assumed that the signs of $b^{j}$ $(j=1,2, \cdots, l)$ do not change in all the rules, i.e., all the $b^{j}$ 's are either positive or negative constants, and this assumption is almost valid for the practical plants. Here, the control objective is to design a fuzzy feedback controller such that it makes the closed-loop system of (8) to be linear stable one. In order to obtain the design objective, this paper utilizes the following Theorem 2.

Theorem 2: Fuzzy feedback linearization of TS fuzzy system

Consider the following fuzzy system as a candidate of the state feedback controller for the plant represented by the TS model (8).

$$
\begin{gathered}
R^{j}: \text { If } x_{1}(t) \text { is } N_{1}^{j}\left(x_{1}\right) \text { and } \cdots \text { and } x_{p}(t) \text { is } N_{p}^{j}\left(x_{p}\right) \\
\text { then } u(t)=-K_{j} \boldsymbol{x}(t)
\end{gathered}
$$

equivalently,

$$
u(t)=\frac{-\sum_{j=1}^{l} \mathrm{rr}_{j}(x(t)) K_{j}}{\sum_{j=1}^{l} \mathrm{r}_{j}(x(t))} x(t)
$$

where, $K_{j}=\left[k_{n}^{j}, k_{n-1}^{j}, \cdots, k_{1}^{j}\right]$, and

$$
\boldsymbol{r}_{j}(\boldsymbol{x}(t))=\coprod_{i=1}^{p} N_{i}^{j}\left(x_{i}(t)\right)
$$

If we choose the design parameters of (11), (12) such that

$$
\begin{gathered}
k_{m}^{j}=\frac{1}{b^{j}}\left(a_{m}^{j}-a_{m}^{d}\right) \quad(m=1,2, \cdots, n) \\
N_{i}^{j}=\sqrt[p]{\left|b^{j}\right|} \cdot M_{i}^{j} \quad(i=1,2, \cdots, p)
\end{gathered}
$$

where $M_{j}^{i}$ are the same fuzzy sets as those of the given plant (8),

then the given nonlinear fuzzy system is transformed into the desired linear system as (15).

$$
\dot{\boldsymbol{x}}(t)=\left|\begin{array}{ccccc}
a_{n}^{d} & a_{n-1}^{d} & \cdots & a_{2}^{d} & a_{1}^{d} \\
1 & 0 & \cdots & 0 & 0 \\
& & \vdots & & \\
0 & 0 & \cdots & 1 & 0
\end{array}\right| \cdot \boldsymbol{x}(t)=A_{d} \boldsymbol{x}(t) .
$$

Proof: Let us first show that

$$
\begin{aligned}
\boldsymbol{k}_{j}(\boldsymbol{x}(t)) & =\prod_{i=1}^{p} N_{i}^{j}\left(x_{i}(t)\right) \\
& =\prod_{i=1}^{p} \sqrt[p]{\left|b^{j}\right|} \cdot M_{i}^{j}\left(x_{i}(t)\right) \\
& =\prod_{i=1}^{p} \sqrt[p]{\left|b^{j}\right|} \cdot \coprod_{i=1}^{p} M_{i}^{j}\left(x_{i}(t)\right) \\
& =\left|b^{j}\right| w_{j}(\boldsymbol{x}(t)) .
\end{aligned}
$$

Then the control input $u$ of (12) is represented in terms of the plant parameters $w_{j}, \quad\left|b^{j}\right|$, and the design parameters $K_{j}$, that is,

$$
u=\frac{-\sum_{j=1}^{l} w_{j}(\boldsymbol{x}(t))\left|b^{j}\right| K_{j}}{\sum_{j=1}^{l} w_{j}(\boldsymbol{x}(t))\left|b^{j}\right|} \boldsymbol{x}(t) .
$$

Substituting the feedback control input $u$ of (16) into the plant equation (8), we obtain the closed-loop system as

$$
\dot{\boldsymbol{x}}=\frac{\sum_{i=1}^{l} w_{i} \cdot A_{i} \boldsymbol{x}-\sum_{i=1}^{l} w_{i} \cdot B_{i}\left(\frac{\sum_{j=1}^{l} w_{j}\left|b^{j}\right| \cdot K_{j}}{\sum_{j=1}^{l} w_{j}\left|b^{j}\right|}\right) \boldsymbol{x}}{\sum_{i=1}^{l} w_{i}} .
$$

Here and after, for the purpose of notational simplicity, we will omit the time notation $t$, and use $w_{j}$ instead of $w_{j}(\boldsymbol{x})$, and use $\sum$ instead of $\sum_{i=1}^{l}$, so long as other notations are not confused with these simplified notations.

Following the obvious manipulations,

$$
\dot{\boldsymbol{x}}=\frac{\left(\sum_{i=1}^{l} w_{i} \cdot A_{i}-\left(\sum_{i=1}^{l} w_{i} \cdot B_{i}\right)\left(\frac{\sum_{j=1}^{l} w_{j}\left|b^{j}\right| \cdot K_{j}}{\sum_{j=1}^{l} w_{j}\left|b^{j}\right|}\right)\right) \boldsymbol{x}}{\sum_{i=1}^{l} w_{i}}
$$

$=\frac{1}{\sum_{i=1}^{l} w_{i}} \cdot\left\{\sum_{i=1}^{l} w_{i} \cdot A_{i}\right.$

$-\left|\begin{array}{c}\sum_{i=1}^{l} w_{i} b^{i} \\ 0 \\ \vdots \\ 0\end{array}\right| \cdot \frac{1}{\sum_{i=1}^{l} w_{j}\left|b^{j}\right|}$

- [ $\left.\left.\sum_{j=1}^{l} w_{j}\left|b^{j}\right| \frac{1}{b^{j}}\left(a_{n}^{j}-a_{n}^{d}\right), \cdots, \sum_{j=1}^{l} w_{j}\left|b^{j}\right| \frac{1}{b^{j}}\left(a_{1}^{j}-a_{1}^{d}\right)\right]\right\} \boldsymbol{x}$

$=\frac{\left(\sum_{i=1}^{l} w_{i} \cdot A_{i}-\left|\begin{array}{c}1 \\ 0 \\ \vdots \\ 0\end{array}\right|\left[\sum_{j=1}^{l} w_{j}\left(a_{n}^{j}-a_{n}^{d}\right), \cdots, \sum_{j=1}^{l} w_{j}\left(a_{1}^{j}-a_{1}^{d}\right)\right]\right.}{\sum_{i=1}^{l} w_{i}} \cdot x$ 


$$
\begin{aligned}
& =\frac{1}{\sum_{i=1}^{L} w_{i}}\left\{\left|\begin{array}{cccc}
\sum_{i=1}^{L} w_{i} a_{n}^{i} & \sum_{i=1}^{1} w_{i} a_{n-1}^{i} & \cdots & \sum_{i=1}^{1} w_{i} a_{1}^{i} \\
1 & 0 & \cdots & 0 \\
0 & 1 & \cdots & 0 \\
0 & 0 & \cdots & 0
\end{array}\right|\right. \\
& \left.-\left|\begin{array}{ccccc}
\sum_{j=1}^{1} w_{j}\left(a_{n}^{j}-a_{n}^{d}\right) & \sum_{j=1}^{1} w_{j}\left(a_{n-1}^{j}-a_{n-1}^{d}\right) & \ldots & \sum_{j=1}^{L} w_{j}\left(a_{1}^{j}-a_{1}^{d}\right) \\
0 & 0 & \ldots & 0 \\
0 & 0 & \ldots & 0 \\
0 & 0 & \ldots & 0
\end{array}\right|\right\} \cdot x \\
& =\frac{\sum_{j=1}^{l} w_{j} \cdot\left|\begin{array}{ccccc}
a_{n}^{d} & a_{n-1}^{d} & \cdots & a_{2}^{d} & a_{1}^{d} \\
1 & 0 & \cdots & 0 & 0 \\
0 & 0 & \vdots & 1 & 0
\end{array}\right|}{\sum_{i=1}^{l} w_{i}} \cdot \boldsymbol{x},
\end{aligned}
$$

we finally achieve the following result.

$$
\dot{\boldsymbol{x}}=\left|\begin{array}{ccccc}
a_{n}^{d} & a_{n-1}^{d} & \cdots & a_{2}^{d} & a_{n}^{1} \\
1 & 0 & \cdots & 0 & 0 \\
& & \vdots & & \\
0 & 0 & \cdots & 1 & 0
\end{array}\right| \boldsymbol{x}=A_{d} \boldsymbol{x}
$$

Remark 3.1: Moreover, if we design the control parameters $a_{m}^{d}$ 's $(m=1,2, \cdots, n)$ of (11), (12) such that the polynomial $h(s)=s^{n}-a_{n}^{d} s^{n-1}-\ldots-a_{1}^{d}$ is Hurwitz (i.e., has all its roots strictly in the LHP), then the closed-loop system (15) is asymptotically stable in the large. The well established linear control techniques such as pole placement and LQG will be helpful to design these parameters. Consequently, by using the FFLC presented in Theorem 2, we can easily construct a fuzzy controller stabilizing the given plant without trial-and-error type of procedures to find a common positive matrix $P$ satisfying Theorem 1, in order to check the stability of the fuzzy feedback system.

\section{Indirect model reference adaptive FFLC}

In some control tasks, the systems to be controlled have constant or slowly-time varying uncertain parameters. Unless such parameter uncertainty is gradually reduced on-line by an appropriate adaptation or estimation mechanism, it may cause inaccuracy or even instability for the control systems. Without continuous redesign of the controller, the initially appropriate controller design may not be able to control the changing plant well. Since the earlier approaches to stabilize fuzzy systems are lack of such considerations, they do not guarantee the stability of system in presence of parameter uncertainty or unmodeled dynamics.

As a control scheme to overcome these problems, we develop an indirect Model Reference Adaptive Fuzzy Control (MRAFC) scheme, where the parameters of the plant are estimated and the estimates, in turn, used to determine the controller parameters at each time $t$. The MRAC schemes based on this approach are referred to as indirect MRAFC schemes since the parameters of fuzzy controller are indirectly estimated using the plant parameters estimates.

Let us consider again the plant represented by the TS model (1)-(2), where $w_{i}(\boldsymbol{x}(t))=\coprod_{j=1}^{p} M_{j}^{i}(\boldsymbol{x}(t))$ and the sign of $b^{j}(j=1,2, \cdots, l)$ are assumed to be known, and $A_{i}, B_{i}$ are unknown. The design objective is to choose the control law such that all signals in the closed-loop plant are bounded and the plant state $\boldsymbol{x}$ tracks the state $\boldsymbol{x}_{m}$ of the reference model

$$
\ddot{\boldsymbol{x}_{m}}=A_{m} \boldsymbol{x}_{m}+B_{m} r
$$

where

$$
A_{m}=\left|\begin{array}{ccccc}
a_{n}^{m} & a_{n-1}^{m} & \cdots & a_{2}^{m} & a_{1}^{m} \\
1 & 0 & \cdots & 0 & 0 \\
& & \vdots & & \\
0 & 0 & \cdots & 1 & 0
\end{array}\right|, B_{m}=\left|\begin{array}{c}
b_{m} \\
0 \\
\vdots \\
0
\end{array}\right| .
$$

The $A_{m}$ is a stable matrix (i.e., has all its eigenvalues in the strictly left-half plane), and the $B_{m}$ and the reference input signal $r$ are chosen so that $\boldsymbol{x}_{m}(t)$ represents the desired state response of the plant.

1. Control law

Recall that the FFLC developed in Section 3

$$
\begin{gathered}
R^{j}: \text { If } x_{1}(t) \text { is } N_{1}^{j}\left(x_{1}\right) \text { and } \cdots \text { and } x_{p}(t) \text { is } N_{p}^{j}\left(x_{p}\right) \\
\text { then } u(t)=-K_{j} x(t)+l_{j} r(t)
\end{gathered}
$$

or equivalently,

$$
u(t)=\frac{\sum_{j=1}^{l} r_{j}(x(t))\left\{-K_{j} x(t)+l_{j} r(t)\right\}}{\sum_{j=1}^{l} r_{j}(x(t))}
$$

with the control parameters

$$
\begin{gathered}
N_{i}^{j}=\sqrt[p]{\left|b^{j}\right|} \cdot M_{i}^{j}(i=1,2, \cdots, p) \\
K_{j}=\left[k_{n}^{j}, k_{n-1}^{j}, \cdots, k_{1}^{j}\right] \\
=\frac{1}{b_{j}}\left[a_{n}^{j}-a_{n}^{m}, a_{n-1}^{j}-a_{n-1}^{m}, \cdots, a_{1}^{j}-a_{1}^{m}\right] \\
l_{j}=\frac{1}{b_{j}} b^{m}
\end{gathered}
$$

could be used to make the closed-loop system of the plant (2) to the desired linear reference model (17)

Therefore, if the plant parameters

$$
A_{i}=\left|\begin{array}{ccccc}
a_{n}^{i} & a_{n-1}^{i} & \cdots & a_{2}^{i} & a_{1}^{i} \\
1 & 0 & \cdots & 0 & 0 \\
& & \vdots & & \\
0 & 0 & \cdots & 1 & 0
\end{array}\right|, B_{i}=\left|\begin{array}{c}
b^{i} \\
0 \\
\vdots \\
0
\end{array}\right|
$$

were known,

the control law

$$
u(t)=\frac{\sum_{j=1}^{l} \mathrm{r}_{j}^{*}(\boldsymbol{x})\left\{-K_{j}^{*} \boldsymbol{x}(t)+l_{j}^{*} r(t)\right\}}{\sum_{j=1}^{l} \mathrm{r}_{j}^{*}(\boldsymbol{x}(t))}
$$

with the parameters

$$
K_{j}^{*}=\frac{1}{b_{j}}\left[a_{n}^{j}-a_{n}^{m}, a_{n-1}^{j}-a_{n-1}^{m}, \cdots, a_{1}^{j}-a_{1}^{m}\right]
$$




$$
\begin{gathered}
l_{j}^{*}=\frac{1}{b_{j}} b^{m} \\
r_{j}^{*}=w_{j}\left|b_{j}\right| \quad(j=1,2, \cdots, l)
\end{gathered}
$$

could be used to meet the control objective.

In the unknown parameter case,

we propose

$$
u(t)=\frac{\sum_{j=1}^{l} \mathrm{r}_{j}(t)\left\{-K_{j}(t) \boldsymbol{x}(t)+l_{j}(t) r(t)\right\}}{\sum_{j=1}^{l} \boldsymbol{r}_{j}(t)}
$$

where $K_{j}(t), l_{j}(t), \quad \boldsymbol{r}_{j}(t)$ are the estimates of $K_{j}^{*}, l_{j}^{*}$, $\mathrm{tr}_{j}^{*}$, respectively. In inderect adaptive control, we evaluate $K_{j}(t), l_{j}(t), r_{j}(t)$ by using the relationship (20) and the estimates $\widetilde{A}_{i}, \quad \breve{B}_{i}$ of the unknown parameters $A_{i}, B_{i}$ $(i=1, \cdots, l)$, as follows:

$$
K_{j}(t)=\frac{1}{\breve{b}_{j}(t)}\left[\quad \check{a}_{n}{ }^{j}(t)-a_{n}^{m}, \quad \check{a}_{n-1}^{j j}(t)-a_{n-1}^{m}, \ldots, \quad \check{a}_{1}^{j}(t)-a_{1}^{m}\right]
$$

$$
\begin{gathered}
l_{j}(t)=\frac{1}{\breve{b}_{j}(t)} b^{m} \\
r_{j}(t)=w_{j}\left|\breve{b}_{j}(t)\right|(j=1,2, \cdots, l)
\end{gathered}
$$

where parameters $\check{a}_{i}^{j}, \quad \breve{b}_{j}(i=1, \cdots, n, j=1, \cdots, l)$ of the parameters $\widetilde{A}_{i}, \quad \breve{B}_{i}$ are generated by an adaptive law that we design.

2. Adaptive law

In order to derive an adaptive law for generating $\overleftarrow{A}_{i}, \breve{B}_{i}$, we rewrite (8) as

$$
\dot{\boldsymbol{x}}=A_{m} \boldsymbol{x}+\frac{\sum_{i=1}^{l} w_{i}(\boldsymbol{x})\left(\left(A_{i^{-}} A_{m}\right) \boldsymbol{x}+B_{i} u\right)}{\sum_{i=1}^{l} w_{i}(\boldsymbol{x})}
$$

and generate $\check{x}$, the estimates of $\boldsymbol{x}$, from

$$
\dot{\ddot{x}}=A_{m} \ddot{\boldsymbol{x}}+\frac{\sum_{i=1}^{l} w_{i}(\boldsymbol{x})\left(\left(\widetilde{A}_{i}(t)-A_{m}\right) \boldsymbol{x}+\widetilde{B}_{i}^{\prime}(t) u\right)}{\sum_{i=1}^{l} w_{i}(\boldsymbol{x})}
$$

which is the same as the reference model, i.e., by substituting (21) and (22) into (24), we have

$$
\ddot{\boldsymbol{x}_{m}}=A_{m} \boldsymbol{x}_{m}+B_{m} r
$$

and

$$
\check{x}=x_{m}
$$

Therefore, the estimation error $\epsilon=\boldsymbol{x}-\check{\boldsymbol{x}}$ is the same as the tracking error $\boldsymbol{e}=\boldsymbol{x}-\boldsymbol{x}_{m}$, and satisfies the differential equation

$$
\dot{\boldsymbol{e}}=A_{m} \boldsymbol{e}+\frac{\sum_{i=1}^{l} w_{i}(\boldsymbol{x})\left(-\widetilde{A_{i}}(t) \boldsymbol{x}-\widetilde{B_{i}}(t) u\right)}{\sum_{i=1}^{l} w_{i}(\boldsymbol{x})}
$$

where $\widetilde{A_{i}} \overline{\bar{\nabla}} \widetilde{A_{i}{ }^{-}} A_{i}, \quad \widetilde{B_{i}} \overline{\bar{\nabla}} \widetilde{B_{i}}-B_{i}$ are the parameter errors.

Rewriting (26), we have

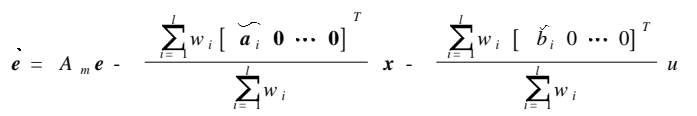

where $\stackrel{\tilde{\boldsymbol{a}}_{i}{ }^{T}}{\overline{\bar{v}}} \widetilde{\boldsymbol{a}}_{i}{ }^{T}-\boldsymbol{a}_{i}{ }^{T}, \breve{b_{i}} \overline{\bar{\nabla}} \breve{b_{i}}-b_{i}$, and $\boldsymbol{a}_{i}{ }^{T}=$ $\left[a_{n}^{i}, a_{n-1}^{i}, \cdots, a_{1}^{i}\right]$

We propose the following function (28) as a Lyapunov function candidate for (27).

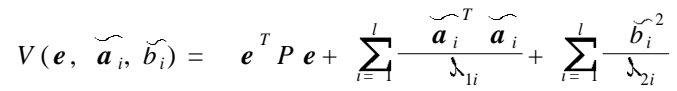

where $\lambda_{1 i}, \lambda_{2 i}>0$ are constants, and $P=P^{T}>0$ is chosen as the solution of the Lyapunov equation

$$
A_{m}^{T} P+P A_{m}=-I
$$

whose existence is guaranteed because we choose the matrix $A_{m}$ so that it can stabilize the reference model. The time derivative $\dot{V}$ of $V$ along the trajectory of (27) is given by

$$
\dot{V}=\dot{e}^{T} P \boldsymbol{e}+\boldsymbol{e}^{T} P \dot{\boldsymbol{e}}+\sum_{i=1}^{l} 2 \frac{\hat{\boldsymbol{a}}_{i}^{T} \widehat{\boldsymbol{a}_{i}}}{\lambda_{1 i}}+\sum_{i=1}^{l} 2 \frac{\widehat{b_{i}} \dot{b_{i}}}{\lambda_{2 i}}
$$

where

$$
\begin{aligned}
& \dot{e}^{T} P \boldsymbol{e}+\boldsymbol{e}^{T} P \dot{\boldsymbol{e}}
\end{aligned}
$$

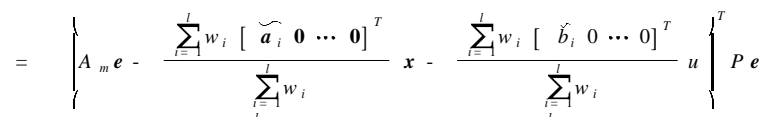

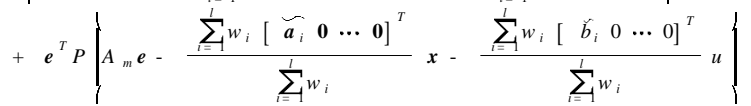

$$
\begin{aligned}
& =\boldsymbol{e}^{T}\left(A_{m}^{T} P+P A_{m}\right) \boldsymbol{e}
\end{aligned}
$$

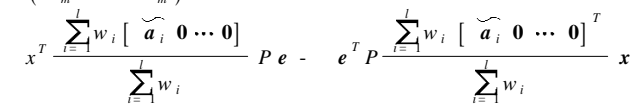

$$
\begin{aligned}
& u \frac{\sum_{i=1}^{t} w_{i}\left[\widehat{b_{i}} 0 \cdots 0\right]}{\sum_{i=1}^{L} w_{i}} P e-e^{T} P \frac{\sum_{i=1}^{L} w_{i}\left[\widehat{b_{i}} 0 \cdots 0\right]^{T}}{\sum_{i=1}^{L} w_{i}} u
\end{aligned}
$$

Therefore,

$$
\begin{aligned}
& \hat{V}=-e^{T} e \\
& \boldsymbol{x}^{T} \frac{\sum w_{i}\left[\widetilde{\boldsymbol{a}_{i}} \mathbf{0} \ldots \mathbf{0}\right]}{\sum w_{i}} P \boldsymbol{e}-\boldsymbol{e}^{T} P \frac{\sum^{w_{i}}\left[\widetilde{\boldsymbol{a}}_{i} \mathbf{0} \ldots \mathbf{0}\right]^{T}}{\sum w_{i}} \boldsymbol{x} \\
& \text { - } u \frac{\sum w_{i}\left[\widehat{b_{i}} 0 \cdots 0\right]}{\sum w_{i}} P e-e^{T} P \frac{\sum w_{i}\left[\widehat{b_{i}} 0 \cdots 0\right]^{T}}{\sum w_{i}} u
\end{aligned}
$$

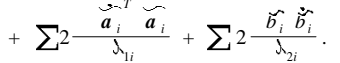

Now, we have

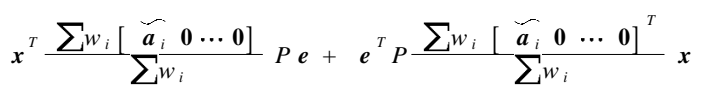




$$
\begin{aligned}
& =\boldsymbol{x}^{T} \frac{\sum w_{i} \widetilde{\boldsymbol{a}_{i}}}{\sum w_{i}} \boldsymbol{p}_{1}{ }^{T} \boldsymbol{e}+\boldsymbol{e}^{T} \boldsymbol{p}_{1} \frac{\sum w^{w_{i}} \stackrel{\widetilde{\boldsymbol{a}}_{i}^{T}}{\sum w_{i}} \boldsymbol{x}}{=2 \boldsymbol{x}^{T} \frac{\sum w_{i} \widetilde{\boldsymbol{a}_{i}}}{\sum w_{i}} \boldsymbol{p}_{1}{ }^{T} \boldsymbol{e}} \\
& =2 \frac{\sum w_{i} \boldsymbol{p}^{T}{ }^{T} \boldsymbol{e} \boldsymbol{x}^{T} \widehat{\boldsymbol{a}_{i}}}{\sum w_{i}}
\end{aligned}
$$

because

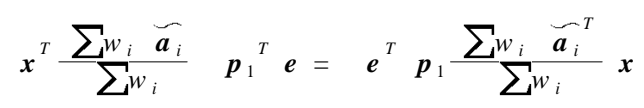

where $\boldsymbol{p}_{1}$ is the first column of $P$.

By using similar manipulation as (32), we have

$$
\begin{aligned}
& u \frac{\sum w_{i}\left[\widehat{b_{i}} 0 \cdots 0\right]}{\sum w_{i}} P \boldsymbol{e}+\boldsymbol{e}^{T} P \frac{\sum w_{i}\left[\widehat{b_{i}} 0 \cdots 0\right]^{T}}{\sum w_{i}} u \\
& =2 u \frac{\sum w_{i} \widetilde{b_{i}}}{\sum w_{i}} \boldsymbol{p}_{1}{ }^{T} \boldsymbol{e} \\
& =2 \frac{\sum w_{i} \widehat{b_{i}}}{\sum w_{i}} \boldsymbol{p}_{1}{ }^{T} \boldsymbol{e} u .
\end{aligned}
$$

By substituting (32) and (33) into (31), we obtain

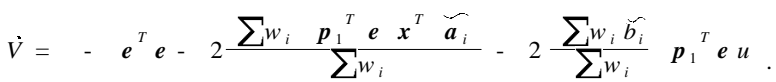

$$
\begin{aligned}
& +\sum 2 \frac{\widetilde{\boldsymbol{a}_{i}} \widetilde{\boldsymbol{a}_{i}}}{\lambda_{1 i}}+\sum 2 \frac{\widetilde{b_{i}} \widetilde{b_{i}}}{\jmath_{2 i}}
\end{aligned}
$$

Since the primary objective of adaptive law is to stabilize the estimation error dynamics, we have to choose the adaptive law such that it makes $\dot{V}$ to be negative.

Hence, for

$$
\begin{aligned}
& \stackrel{\tilde{\boldsymbol{a}}_{i}^{T}}{T}=\stackrel{\dot{\boldsymbol{a}}_{i}^{T}}{T}=\lambda_{1 i}-\frac{w_{i}}{\sum w_{i}} \boldsymbol{p}_{1}{ }^{T} \boldsymbol{e} \boldsymbol{x}^{T} \\
& \dot{b_{i}}=\dot{b_{i}}=\lambda_{2 i}-\frac{w_{i}}{\sum w_{i}} \quad \boldsymbol{p}_{1}{ }^{T} \boldsymbol{e} u
\end{aligned}
$$

we have

$$
\dot{V}=\quad-e^{T} e<0
$$

which implies that $\boldsymbol{e}, \quad \check{\boldsymbol{a}}_{i}, \breve{b}_{i} \in \Phi_{\infty}$.

From (36), we conclude that because $V$ is bounded from below and is nonincreasing with time, it has a limit, i.e.,

$$
\lim _{t \rightarrow \infty} V\left(\boldsymbol{e}(t), \widetilde{A_{i}}(t), \widetilde{B_{i}}(t)\right)=V_{\infty}<\infty
$$

From (36) and (37), it follows that

$$
\vec{b}^{\infty} e^{T} \boldsymbol{e} d \varsigma=-\hat{l}_{0}^{\infty} \dot{V} d \varsigma=\left(V_{0}-V_{\infty}\right)
$$

where

$$
V_{0}=V\left(\boldsymbol{e}(0), \widetilde{A}_{i}(0), \widetilde{B}_{i}(0)\right)
$$

which implies that $e \in \Phi_{2}$.

Furthermore, $x_{m}, \boldsymbol{e} \in \mathscr{\delta}_{\infty}$ imply that $\boldsymbol{x} \in \mathscr{\delta}_{\infty}$. The boundedness of $u$, however, cannot be established unless we show that $K_{j}(t), l_{j}(t), r_{j}(t)$ are bounded. The boundedness of $\boldsymbol{r}_{j}(t)$ is easily established from $r_{j}(t)=$ $w_{j}(\boldsymbol{x}(t))\left|\breve{b}_{j}(t)\right|, \quad 0<w_{j}(\boldsymbol{x}(t))<1 \quad$ and $\quad \breve{b}_{j} \in \Phi_{\infty}$. However, the boundedness of $1 / \breve{b}_{j}$ and therefore of $K_{j}(t), l_{j}(t)$ cannot be guaranteed by the adaptive law (35) because (35) may generate estimates $\breve{b}_{j}(t)$ arbitrarily close or even equal to zero. The requirement that $\breve{b}_{j}^{\prime}(t)$ is bounded away from zero is a controllability condition for the estimated plant that the control law (21) is designed for. One method for avoiding $\breve{b}_{j}(t)$ going through zero is to modify the adaptive law for $\breve{b}_{j}(t)$ so that adaptation takes place in a closed subset of $R^{1}$ which does not include the zero element. Such a modification is achieved by using the following a priori knowledge:

The $\operatorname{sgn}\left(b_{j}\right)$ and a lower bound $b_{j 0}>0$ for $\left|b_{j}\right|$ is known for all $j=1, \cdots, l$

Applying the projection method with the constraint $\breve{b_{j}} \operatorname{sgn}\left(b_{j}\right)>b_{j 0}$ to the adaptive law (35), we obtain

$$
\begin{aligned}
& \tilde{\boldsymbol{a}}_{i}^{T}=\lambda_{1 i}-\sum_{i} w_{i} p_{1}{ }^{T} \boldsymbol{e} \boldsymbol{x}^{T} \\
& \grave{b}_{i}=\left\{\begin{array}{lll}
\mathfrak{l}_{2 i}-\frac{w_{i}}{\sum w_{i}} \quad \boldsymbol{p}_{1}{ }^{T} \boldsymbol{e} u & \text { if }\left|\breve{b}_{i}\right|>b_{i 0} \text { or } \\
& & \text { if }\left|\breve{b}_{i}\right|=b_{i 0} \text { and } \\
& \boldsymbol{p}_{1}{ }^{T} \boldsymbol{e} u \operatorname{sgn}\left(b_{i}\right)>0 \\
& \text { otherwise }
\end{array}\right.
\end{aligned}
$$

where $\breve{b_{i}}(0)$ is chosen so that $\breve{b_{i}}(0) \operatorname{sgn}\left(b_{i}\right)>b_{i 0}$.

3. Analys is

Using arguments discussed during the derivation of the adaptation law for the TS model, we establish the following theorem which shows the properties of the indirect MRAFC scheme. The control law (21) together with the adaptive law (39) guarantee the boundedness of all signals in the closed-loop system. In addition, the plant state $x$ tracks the state of the reference model $\boldsymbol{x}_{m}$ asymptotically with time for any bounded reference input signal $r$.

Theorem 3: Stability of the indirect MRAFC system

Consider the plant model (2) and the reference model (17) with the control law (21) and adaptive law (39). Assume that the reference input $r$ and the state $\boldsymbol{x}_{m}$ of the reference model are uniformly bounded. Then the control law (21) together with the adaptive law (39) guarantee that

i) $K_{i}(t), l_{i}(t), \boldsymbol{r}_{i}(t), e(t)$ are bounded for all $i=1, \cdots, l$

ii) $\boldsymbol{e}(t) \rightarrow 0$ as $t \rightarrow \infty$

iii) $\dot{\ddot{a}}_{i}, \quad \ddot{b}_{i} \rightarrow 0$ as $t \rightarrow \infty$.

Proof: It follows from (39) that if $\breve{b_{i}}(0) \operatorname{sgn}\left(b_{i}\right)>b_{i 0}$ for all $i=1, \cdots, l$, then whenever $\breve{b_{i}}(t) \operatorname{sgn}\left(b_{i}\right)=\left|\overleftarrow{b_{i}}(t)\right|$ becomes equal to $b_{i 0}$ we have $\check{b}_{i}^{\circ} \breve{b}_{i}>0$ which implies that $\left|\widehat{b_{i}}(t)\right|>b_{i 0}$ for all $t>0$. Futhermore the time derivative of (28) along the trajectory of (27) and (39) satisfies 


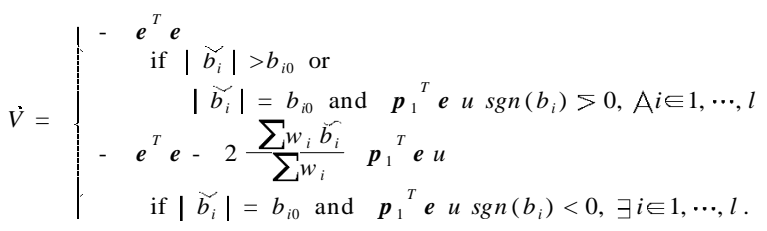

Now for $\left|\breve{b_{i}}(t)\right|=b_{i 0}$, we have $\left(\breve{b_{i}}(t)-b_{i}\right) \operatorname{sgn}\left(b_{i}\right)<0$. Therefore, for $\left|\breve{b}_{i}(t)\right|=b_{i 0}$ and $\quad \boldsymbol{p}_{1}{ }^{T} \boldsymbol{e} u \operatorname{sgn}\left(b_{i}\right)<0$, we have $\widetilde{b_{i}} \boldsymbol{p}_{1}{ }^{T} \boldsymbol{e} u=\left(\widehat{b_{i}}(t)-b_{i}\right) \operatorname{sgn}\left(b_{i}\right)\left(\boldsymbol{p}_{1}{ }^{T} \boldsymbol{e} u \operatorname{sgn}\left(b_{i}\right)\right)>0$ which implies that

$$
\dot{V}<-\boldsymbol{e}^{T} \boldsymbol{e}<0, \quad \mathrm{~A}^{t}>0 .
$$

Therefore, the function $V$ given by (28) is the Lyapunov function for the system (27), (39), since $u, x$ in (27) can be expressed in terms of $\boldsymbol{e}$ and $\boldsymbol{x}_{m}$ where $\boldsymbol{x}_{m}$ is a bounded function of time.

Hence the equilibrium $\boldsymbol{e}_{e}, \quad \check{\boldsymbol{a}}_{i}, \breve{b}_{i}$ is uniformly stable and $\boldsymbol{e}, \quad \check{\boldsymbol{a}}_{i}, \breve{b}_{i} \in \Phi_{\infty}$. The boundedness of $K_{i}(t), l_{i}(t), \boldsymbol{r}_{i}(t)$ is directly established from the boundedness of $\check{\boldsymbol{a}}_{i}, \breve{b}_{i}$ and (23), (39b).

Now, let us show that $\boldsymbol{e} \in \Upsilon_{2}$. From (28) and (41), we conclude that because $V$ is bounded from below and is nonincreasing with time, it has a limit, i.e.,

$$
\lim _{t \rightarrow \infty} V\left(\boldsymbol{e}_{1}(t), \widehat{\boldsymbol{a}_{i}}(t), \widetilde{b_{i}}(t)\right)=V_{\infty}<\infty .
$$

From (B.2) and (B.3), it follows that

$$
\hat{l}_{0}^{\infty} e^{T}(s) \boldsymbol{e}(s) d s<-\hat{l}_{0}^{\infty} \dot{V} d s=\left(V_{0}-V_{\infty}\right)
$$

where, $V_{0}=V\left(\boldsymbol{e}(0), \widetilde{a_{i}}(0), \widehat{b_{i}}(0)\right)$, i.e., $\boldsymbol{e} \in \Phi_{\delta_{2}}$. Because $u, \widehat{\boldsymbol{a}_{i}}, \widetilde{b_{i}}, \boldsymbol{x} \in \Phi_{\infty}$, it follows from (4.12) that $\dot{\boldsymbol{e}} \in \Phi_{\infty}$, which, together with $\boldsymbol{e} \in \mathscr{\delta}_{2}$, implies that $\boldsymbol{e}=\boldsymbol{x}$ $\boldsymbol{x}_{m} \rightarrow \mathbf{0}$ as $t \rightarrow \infty$ and therefore $\dot{\boldsymbol{a}}_{i}, \ddot{b}_{i} \rightarrow 0$ as $t \rightarrow \infty$.

\section{Implementation}

Since all the quantities for generating the control input $u$ are known, available for measurement, or estimated, the control law (21) for the indirect MRAFC of the TS fuzzy model (2) can be implementable. The overall scheme of the indirect MRAFC for the plant (2) is shown in Fig. 1. The reference model is used to specify the ideal response that the fuzzy control system should follow.

The plant is assumed to contain unknown parameters, but its structure is known. The fuzzy controller is constructed from fuzzy systems whose parameters are adjusted indirectly from the estimates. The adaptation law adjusts the plant parameters $\check{\boldsymbol{a}}_{i}, \breve{b}_{i}$ on-line such that the state $\boldsymbol{x}$ of plant tracks the state $\boldsymbol{x}_{m}$ of reference model, which allows plant output to follow the reference model output.

Now, we summarizes the controller design procedure as follows.

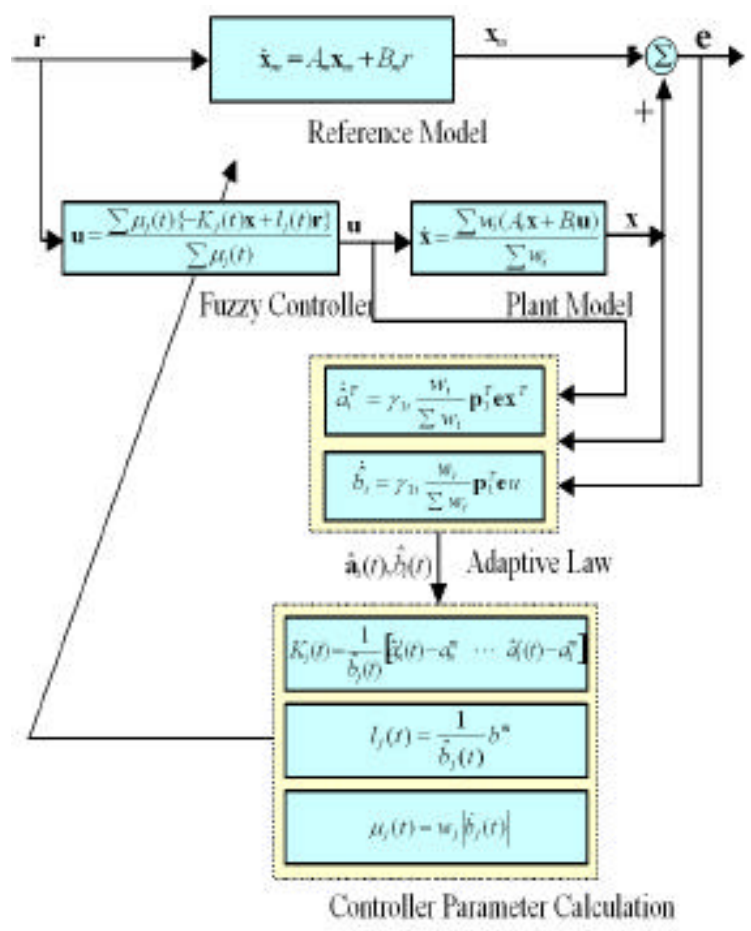

Fig. 1. The overall scheme of indirect MRAFC.

Step 1: Select a stable reference model the plant states have to follow as (17)

Step 2: Design control rule with appropriate control parameters as (18) and (19)

Step 3: Choose a symmetric positive definite matrix $\boldsymbol{P}$ satisfying (29) and get its first column vector $\boldsymbol{p}_{1}$

Step 4: Design a adaptive law as (39).

\section{Simulation and results}

\section{Design example}

The design concept of FFLC is illustrated through an example in this subsection. The example is the problem of balancing and swing-up of an inverted pendulum on a cart shown in Fig. 2. The dynamic equations of motion for the pendulum are given as followings[12][13]:

$$
\begin{gathered}
\dot{x}_{1}=x_{2} \\
\dot{x_{2}}=\frac{g \sin \left(x_{1}\right)-a m l x_{2}^{2} \sin \left(2 x_{1}\right) / 2-a \cos \left(x_{1}\right) u}{4 l / 3-a m l \cos ^{2}\left(x_{1}\right)}
\end{gathered}
$$

where $x_{1}$ denotes the angle (in radian) of the pendulum from the vertical, and $x_{2}$ is the angular velocity. $g=9.8 \mathrm{~m} / \mathrm{s}^{2}$ is the gravity constants, $m$ is the mass of the pendulum, and $u$ is the force applied to the cart (in Newtons). $a=1 /(m+M)$.

The control objective in this example is to balance the inverted pendulum for the approximate range $x_{1} \in(-\Downarrow 2, \Downarrow 2)$. To apply the FFLC design approach, we use the TS fuzzy model representing the dynamics of the inverted pendulum (44). 


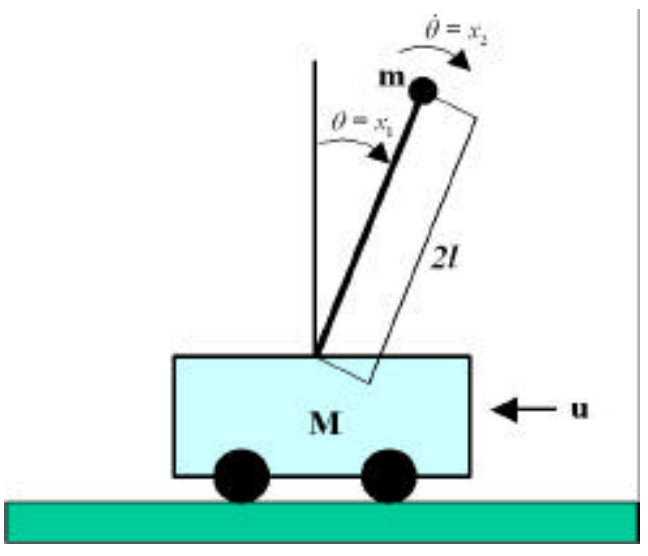

Fig. 2. The inverted pendulum system.

\section{TS fuzzy model representation}

We use the TS fuzzy model derived in [12] as a representation of the system (44). In [12], the dynamic equations are approximated by the following two-rule TS fuzzy model.

$$
\begin{aligned}
& \text { Rule 1: If } x_{1} \text { is } M_{1}^{1} \text { Then } \dot{\boldsymbol{x}}=A_{1} \boldsymbol{x}+B_{1} u \\
& \text { Rule 2: If } x_{1} \text { is } M_{1}^{2} \text { Then } \dot{\boldsymbol{x}}=A_{2} \boldsymbol{x}+B_{2} u
\end{aligned}
$$

where $\boldsymbol{x}^{T}=\left[x_{2}, x_{1}\right]$ and

$A_{1}=\left|\begin{array}{cc}0 & \frac{g}{4 l / 3-a m l} \\ 1 & 0\end{array}\right|, \quad B_{1}=\left|\begin{array}{c}-\frac{a}{4 l / 3-a m l} \\ 0\end{array}\right|$

$A_{2}=\left|\begin{array}{cc}0 & \frac{2 g}{\Psi\left(4 l / 3-a m l B^{2}\right)} \\ 1 & 0\end{array}\right|, \quad B_{2}=\left|-\frac{a b}{4 l / 3-a m l B^{2}}\right|$

and $B=\cos 88^{\circ}$. Membership functions for fuzzy sets $M_{1}^{1}, M_{1}^{2}$ are shown in Fig. 3. In this example and the following simulation, by choosing $m=2.0 \mathrm{~kg}, M=8.0 \mathrm{~kg}$, $2 l=1 \mathrm{~m}$, we obtain

$$
\begin{array}{ll}
A_{1}=\left[\begin{array}{cc}
0 & 17.29 \\
1 & 0
\end{array}\right], & B_{1}=\left[\begin{array}{c}
-0.1765 \\
0
\end{array}\right] \\
A_{2}=\left[\begin{array}{cc}
0 & 9.36 \\
1 & 0
\end{array}\right], & B_{2}=\left[\begin{array}{c}
-0.0052 \\
0
\end{array}\right] .
\end{array}
$$

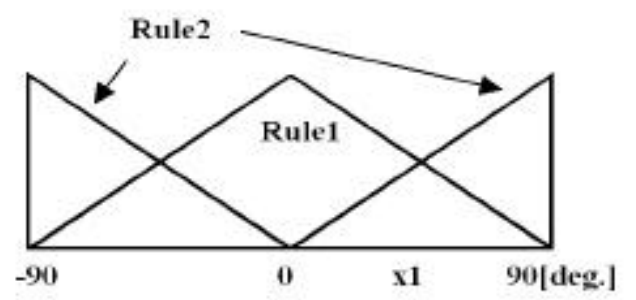

Fig. 3. Membership functions for $M_{1}^{1}, M_{1}^{2}$.

\section{Design of MRAFC and simulation}

As the first design step, we construct the reference model by choosing the appropriate eigenvalues of the closed-loop system. Choosing the closed-loop eigenvalues as
[- 2, - 2], we obtain $A_{m}=\left[\begin{array}{cc}-4 & -4 \\ 1 & 0\end{array}\right]$ and we also choose $B_{m}=\left[\begin{array}{l}1 \\ 0\end{array}\right]$. And then, following the design technique of MRAFC in section 4, we can construct the fuzzy feedback controller as followings:

$$
\text { Rule1: If } x_{1} \text { is } N_{1}^{1} \text { Then } u=-K_{1}(t)\left[\begin{array}{l}
x_{2} \\
x_{1}
\end{array}\right]+l_{1}(t) r(t)
$$

Rule2: If $x_{1}$ is $N_{1}^{2}$ Then $u=-K_{2}(t)\left[\begin{array}{l}x_{2} \\ x_{1}\end{array}\right]+l_{2}(t) r(t)$

where,

$$
\begin{aligned}
& K_{1}(t)=\left[k_{2}^{1}(t), k_{1}^{1}(t)\right]=\left[\frac{1}{\breve{b}^{1}(t)}\left(\widetilde{a_{2}^{1}}(t)-a_{2}^{m}\right), \frac{1}{\widetilde{b}^{1}(t)}\left(\widetilde{a_{1}^{1}}(t)-a_{1}^{m}\right)\right] \\
& K_{2}(t)=\left[k_{2}^{2}(t), k_{1}^{2}(t)\right]=\left[\frac{1}{\widetilde{b^{2}}(t)}\left(\widetilde{a_{2}^{2}}(t)-a_{2}^{m}\right), \frac{1}{\widetilde{b^{2}}(t)}\left(\widetilde{a} a_{1}^{2}(t)-a_{1}^{m}\right)\right] \\
& N_{1}^{1}=\left|\widetilde{b^{1}}(t)\right| \cdot M_{1}^{1}, \quad N_{1}^{2}=\left|\widetilde{b^{2}}(t)\right| \cdot M_{1}^{2} .
\end{aligned}
$$

We can obtain the control input $u(t)$ by inferring the fuzzy control law (46) as follows.

$$
u(t)=\frac{\sum_{j=1}^{2} \mathrm{r}_{j}(\boldsymbol{x}(t))\left\{-K_{j} x(t)+l_{j} r(t)\right\}}{\sum_{j=1}^{2} \mathrm{r}_{j}(\boldsymbol{x}(t))}
$$

where,

$$
\begin{array}{ll}
\mathfrak{r}_{1}(t)=w_{1}\left|\widetilde{b}_{1}(t)\right|, \quad & \boldsymbol{r}_{2}(t)=w_{2}\left|\widetilde{b}_{2}(t)\right| \\
l_{1}(t)=\frac{1}{\widetilde{b_{1}}(t)} b_{m}, & l_{2}(t)=\frac{1}{\bar{b}_{2}(t)} b_{m} .
\end{array}
$$

The plant parameters $\check{\boldsymbol{a}}_{i}, \breve{b}_{i}$ are adjusted on-line by adaptive law (39). where the adaptation rates $\lambda_{11}=50$, and $\lambda_{21}=0.5$ are used and $p_{1}=[0.1562,0.1250]^{T}$, the column of the positive definite matrix $\mathrm{P}$ is obtained by solving the Lyapunov equation. And the lower bound of $b_{i 0}$ is selected as $b_{10}=0.1$ and $b_{20}=0.003$.

This design example show us that the design procedures are simple and intuitively understandable. No additional procedure for checking the stability is required except the procedure for determining the eigenvalues of the closed-loop system showing the desired characteristics. Theorem 3 indicates that the stability of the TS fuzzy system is guaranteed by the appropriate compensation of MRAFC.

The MRAFC designed in this example is simulated and the effects of parametric perturbations on their performance are examined. In this simulation, the MRAFC is applied to the regulating and tracking control problems of the plant. By applying the controller to not only the TS model (44) but also the original model (45), we access the effectiveness of the MRAFC. Table 1 shows the values of varied plant parameters.

The plant parameters are fixed to the initial values for 10 seconds and they are varied linearly to the final values at 20 second. After they reached the final values, they are fixed to the final values. In this simulation, it was assumed 
that the simulated plant model and reference model have their initial states respectively as

$$
\begin{aligned}
& \text { plant model : } x_{10}=-\frac{\mathbb{}}{6} \mathrm{rad}, \quad x_{20}=0 \mathrm{rad}, \\
& \text { reference model }: x_{10}^{m}=-\frac{\square}{9} \mathrm{rad}, \quad x_{20}^{m}=0 \mathrm{rad}
\end{aligned}
$$

Fig. 4 and Fig. 5 show the simulation results of regulating control of respective fuzzy model and original model. The regulation errors of both the control systems are illustrated in Fig. 6. Figure 7 and Figure 8 presents the tracking control of MRAFC and the tracking errors are shown in Fig. 9. It is clear form the results that the MRAFC designed in this example is robust to plant parameter perturbations in both tracking and regulating control.

Table 1. Variation of plant parameters.

\begin{tabular}{|c|c|c|c|c|}
\hline \multirow{2}{*}{} & \multicolumn{5}{|c|}{ Values of plant parameters } \\
\cline { 2 - 5 } & $A_{1}$ & $A_{2}$ & $B_{1}$ & $B_{2}$ \\
\hline $\begin{array}{c}\text { Initial } \\
\text { value }\end{array}$ & {$\left[\begin{array}{cc}0 & 17.29 \\
1 & 0\end{array}\right]$} & {$\left[\begin{array}{cc}0 & 9.36 \\
1 & 0\end{array}\right]$} & {$\left[\begin{array}{c}-0.1765 \\
0\end{array}\right]$} & {$\left[\begin{array}{c}-0.0052 \\
0\end{array}\right]$} \\
\hline $\begin{array}{c}\text { Final } \\
\text { value }\end{array}$ & {$\left[\begin{array}{cc}0 & 20 \\
1 & 0\end{array}\right]$} & {$\left[\begin{array}{cc}0 & 11 \\
1 & 0\end{array}\right]$} & {$\left[\begin{array}{c}-0.2 \\
0\end{array}\right]$} & {$\left[\begin{array}{c}-0.01 \\
0\end{array}\right]$} \\
\hline
\end{tabular}

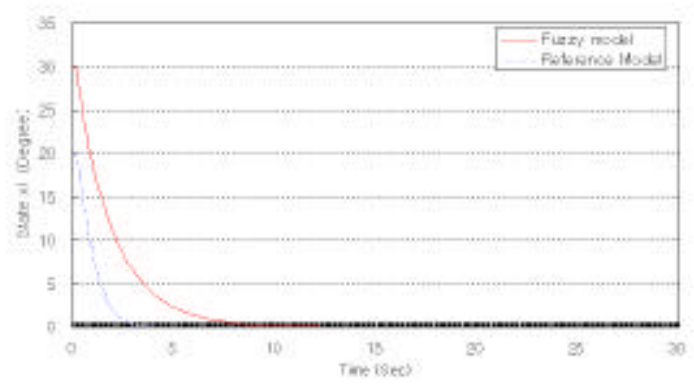

Fig. 4. Regulating control applied to the fuzzy model.

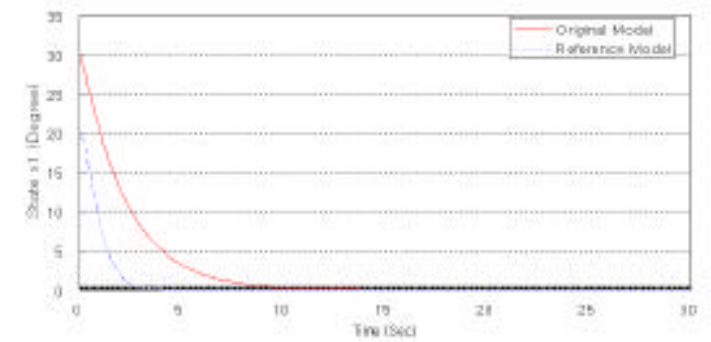

Fig. 5. Regulating control applied to the original model.

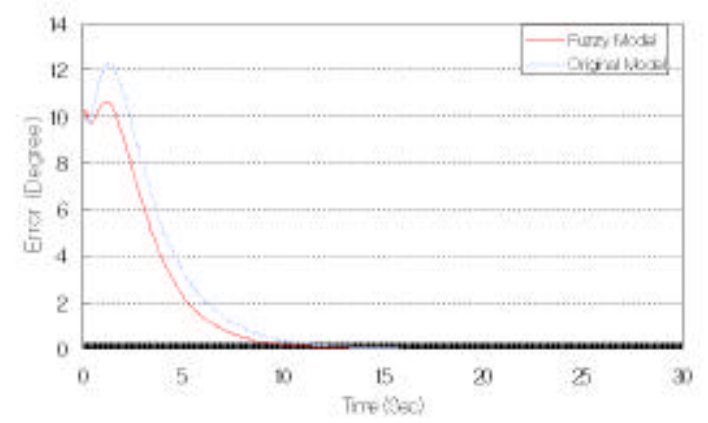

Fig. 6. Regulating error applied to the fuzzy model and original model.

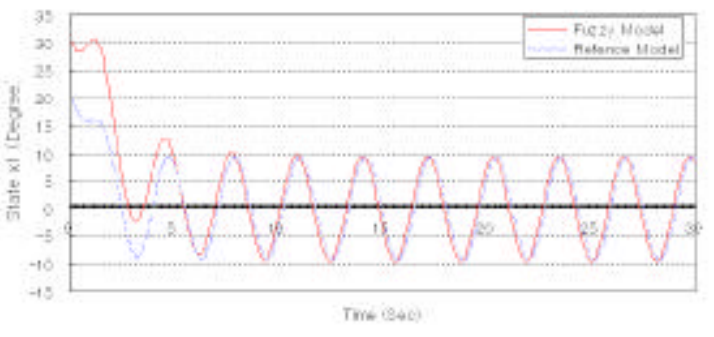

Fig. /. I racking control applied to the tuzzy model.

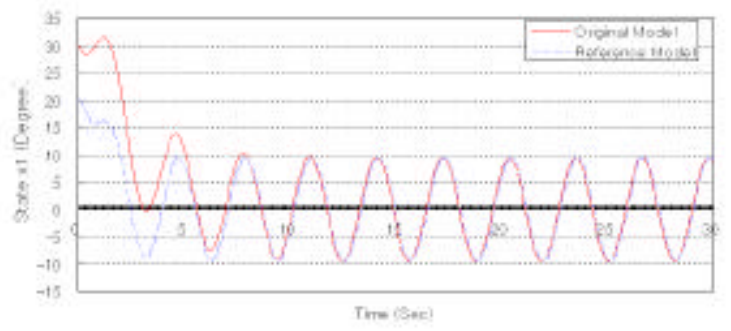

rig. ஓ. I racking control appıred to the original model.

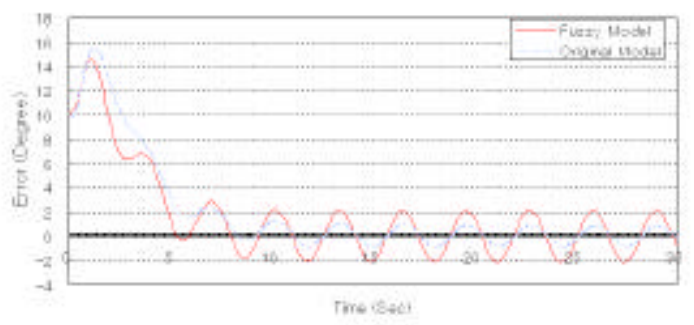

Fig. y. I racking error applied to the ruzzy model and original model.

\section{Conclusions}

In this paper, we have developed an indirect model reference adaptive control scheme for the SISO Takagi-Sugeno model whose structure is assumed to be known but the parameters unknown. The fuzzy controller is constructed from fuzzy feedback linearization controller whose parameters are adjusted indirectly from the estimates of plant parameters. The adaptation law adjusts the controller parameters on-line so that the plant output tracks the reference model output. The developed control law and adaptive law guarantee the boundedness of all signals in the closed-loop system and ensure that the plant state tracks the state of the reference model asymptotically with time for any bounded reference input signal. Since all the quantities for generating the control input are known, available for measurement, or estimated. the developed control law for the indirect MRAFC of the TS fuzzy model can be implementable. Development of a fuzzy feedback linearization scheme for MIMO systems is recommended as the future works. The fuzzy feedback linearization control of MIMO system will make the design and implementation of the adaptive fuzzy system easy. 


\section{References}

[1] K. Astrom and B. Wittenmark, Adaptive Control, Addison Wesley, pp. 343-359, 1988.

[2] P. A. Ioannou and J. Sun, Robust Adaptive Control, PrenticeHall International Editions, 1996.

[3] S.-W. Kim, Y.-W. Cho, and M. Park, "A multirule-basecontroller using the robust property of a fuzzy controller and its design method," IEEE Trans. on Fuzzy Systems, vol. 4, no. 3, pp. 315-327, Aug., 1996.

[4] Y.-W. Cho, S.-H. Lee, K.-J. Kim, and M. Park, "Analysis and design of robust fuzzy feedback linearization controller based on Takagi-Sugeno model," Proc. of IIZUKA 98 , pp. 434-437.

[5] L. K. Wong, F. H. F. Leung, and P. K. S. Tam, "Stability design of TS model based fuzzy systems," in Proc. of 6th IEEE Int. Conf. on Fuzzy Systems, pp. 83-86, 1997.

[6] M. Maeda and S. Murakami, "A self-tuning fuzzy controller," Fuzzy Sets and Systems, vol. 51, pp. 29-40, 1992.

[7] C. S. Ting, T. H. S. Li, and F. C. Kung, "An approach to systematic design of fuzzy control system," Fuzzy Sets and Systems, vol. 77, pp. 151-166, 1996.

[8] L. -X. Wang, A Course in Fuzzy systems and Control, Prentice-Hall International, Inc., 1997.

[9] R. M. Tong, "Some properties of fuzzy feedback systems," IEEE Trans. on Systems, Man and Cybernetics, vol. 10, no. 6, pp. 327-330, 1980.

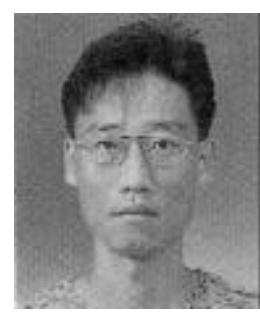

\section{Young-Wan Cho}

He was born in Hamyang, Korea, in 1968. He received the B.S., M.S., and $\mathrm{Ph} . \mathrm{D}$. degrees in electronic engineering from Yonsei University, Seoul, Korea, in 1991, 1993, and 1999, respectively. $\mathrm{He}$ is currently a researcher in Samsung Electronics. His research interests include robotics and automation, machine vision, intelligent control and nonlinear control.
[10] T. Takagi and M. Sugeno, "Fuzzy identification of systems and its applications to modeling and control," IEEE Trans. Systems, Man, and Cybernetics, vol. 15, no. 1, pp. 116-132, 1985.

[11] K. Tanaka and M. Sano, "A robust stabilization problem of fuzzy control systems and its application to backing up control of a truck-trailer," IEEE Trans. Fuzzy Systems, vol. 2, no. 2, pp. 119-133, 1994.

[12] H. O. Wang, K. Tanaka, and M. F. Griffin, "An approach to fuzzy control of nonlinear systems: Stability and design issues, "2 IEEE Trans. on Fuzzy Systems, vol. 4, no. 1, pp. 14-23, Feb., 1996.

[13] L. X. Wang, Adaptive Fuzzy Systems and Control: Design and stability analysis, Englewood Cliffs, NJ: Prentice-Hall, 1994.

[14] K. Tanaka, Advanced Fuzzy Control. Tokyo, Japan: Kyouritu Pub., 1994 (in Japanese).

[15] K. Tanaka and M. Sugeno, "Stability analysis and design of fuzzy control systems," Fuzzy Sets and Systems, vol. 45, no. 2, pp. 135-156, 1992.

[16] H. -J. Kang, C. Kwon, H.-J. Lee, and M. Park, "Robust stability analysis and design method for the fuzzy feedback linearization regulator," IEEE Trans. on Fuzzy Systems, vol. 6, no. 4, pp. 464-472, Nov., 1998.

[17] Spooner, J. T. and Passino, K. M., "Stable adaptive control using fuzzy systems and neural networks," IEEE Trans. on Fuzzy Systems, vol. 4, no. 3, pp. 339-359, Aug., 1996.

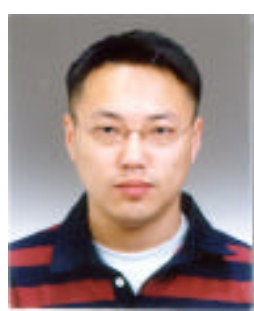

\section{Chang-Woo Park}

He was born in Seoul, Korea, in 1973. $\mathrm{He}$ received the B.S. degree in electronics from Korea University and M.S. degree in electronics from Yonsei University, Seoul, Korea, in 1997 and 1999, respectively. $\mathrm{He}$ is currently working toward the Ph.D. degree in electrical and computer engineering at Yonsei University, Seoul, Korea. His current research interests include fuzzy control theory, nonlinear control theory and robot vision system. 


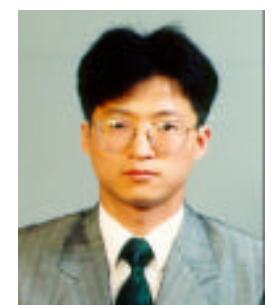

Ki-Chul Lee

He received the B.S., M.S., and Ph.D. degrees in electronic engineering from Yonsei University, Seoul, Korea, in 1991, 1993, and 2000, respectively. His research interests include nonlinear control, robotics and intelligent control.

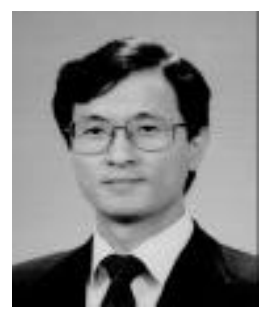

\section{Mignon Park}

He received the B.S. and M.S. degrees in electronics from Yonsei University, Seoul, Korea, in 1973 and 1977, respectively, and the $\mathrm{Ph}$. D. degree from the University of Tokyo, Tokyo, Japan, in 1982. From 1977 to 1982, he was a Researcher at the Institute Biomedical Engineering, University of Tokyo, Japan. In 1982 he was a Researcher at Massachusetts Institute of Technology, Cambridge, MA, and at the University of California, Berkely. He was a Visiting Researcher in Robotics Division, Mechanical Engineering Laboratory (MEL) and Ministry of International Trade and Industry (MITI), Tsukuba, Japan, from 1986 to 1987. Since 1982, he has been a Professor in the Electrical and Computer Department of Yonsei University. His research interests include fuzzy control and application engineering, robotics, and fuzzy biomedical system, etc. Dr. Park was a Program Chairman of the Korea-Japan Joint Conference on Fuzzy Systems and Engineering in 1992, and the Vice-Chair of IEEE-FUZZ99 in 1999. He was the chairman of Korea-Japan Joint Workshop in 2000. He is a member of the Japanese Society for Fuzzy Theory and Systems and the Japanese Society for Biomedical Fuzzy Systems, among others. 